\title{
Differential effects of insulin and dexamethasone on pulmonary surfactant-associated genes and proteins in A549 and H441 cells and lung tissue
}

\author{
ZDENEK RUCKA ${ }^{1}$, PETR VANHARA ${ }^{2}$, IRENA KOUTNA ${ }^{1}$, LENKA TESAROVA ${ }^{1}$, \\ MICHAELA POTESILOVA ${ }^{1}$, STANISLAV STEJSKAL ${ }^{1}$, PAVEL SIMARA ${ }^{1}$, JAN DOLEZEL $^{3}$, \\ VACLAV ZVONICEK ${ }^{4}$, OLDRICH COUFAL ${ }^{5}$ and IVAN CAPOV ${ }^{3}$ \\ ${ }^{1}$ Centre for Biomedical Image Analysis, Faculty of Informatics, Masaryk University, 60200 Brno; \\ ${ }^{2}$ Department of Histology and Embryology, Faculty of Medicine, Masaryk University, 62500 Brno; \\ ${ }^{3} 1$ st Department of Surgery and ${ }^{4}$ Department of Anesthesiology and Intensive Care, St. Anne's University Hospital Brno, \\ 65691 Brno; ${ }^{5}$ Department of Surgical Oncology, Masaryk Memorial Cancer Institute, 65653 Brno, Czech Republic
}

Received February 10, 2013; Accepted April 10, 2013

DOI: $10.3892 /$ ijmm.2013.1363

\begin{abstract}
In this study, the effects of insulin and dexamethasone on the expression and mRNA transcription of 4 pulmonary surfactant-associated proteins [surfactant protein (SFTP)A, SFTPB, SFTPC and SFTPD] were examined. The commercially available cell lines, A549 and H441, were used as acceptable models of lung surfactant-producing cells. Subsequently, the effects of insulin on the expression of surfactant-associated proteins were examined in patients with lung adenocarcinoma during lung resection. Our results demonstrated the inhibitory effects of insulin on the transcription of the SFTPB, SFTPC and SFTPD genes in H441 cells and the SFTPB gene in A549 cells. Treatment with insulin significantly decreased the protein expression of SFTPA1 and SFTPA2 in the H441 cells and that of proSFTPB in the A549 cells. Dexamethasone promoted the transcription of the SFTPB, SFTPC and SFTPD genes in the A549 and H441 cells and reduced the transcription of the SFTPA1 and SFTPA2 genes in the H441 cells (SFTPA mRNA expression was not detected in A549 cells). Furthermore, we demonstrated that the mRNA levels of the selected genes were significantly lower in the cell lines compared to the lung tissue. A549 and H441 cells represent similar cell types. Yet, in our experiments, these cells reacted differently to insulin and/or dexamethasone treatment, and the mRNA levels of their main protein products, surfactant-associated proteins,
\end{abstract}

Correspondence to: Dr Irena Koutna, Centre for Biomedical Image Analysis (CBIA), Faculty of Informatics, Masaryk University, ILBIT A3, Kamenice 5, 62500 Brno, Czech Republic

E-mail: cbia.muni@gmail.com; qkoutna@fi.muni.cz

Key words: insulin, dexamethasone, surfactant, lung carcinoma, A549, H441 were significantly lower than those in real tissue. Therefore, the results obtained in this study challenge the suitability of A549 and H441 cells as models of type II pneumocytes and Clara cells, respectively. However, we successfully demonstrate the possibility of studying the effects of insulin on pulmonary surfactant-associated genes and proteins in patients with lung adenocarcinoma.

\section{Introduction}

From the treatment of diabetes to experimental therapy in critically ill patients, insulin is a widely used drug affecting a number of metabolic pathways in human tissue (1-5). In the present study, we characterized the effects of insulin on pulmonary surfactant-associated genes and proteins (SFTPs). The expression levels of 5 genes (SFTPA1, SFTPA2, SFTPB, SFTPC and SFTPD) and their 4 protein products (SFTPA, SFTPB, SFTPC and SFTPD) were examined in 2 cell lines, A549 and H441. Subsequently, we determined the feasibility of performing these measurements in patients with lung adenocarcinoma.

The A549 and H441 cell lines are commonly used as models of lung surfactant-producing cells. A549 is a human lung adenocarcinoma cell line which was established in 1972. A549 cells have been used as a model of type II pneumocytes, demonstrating typical morphology and ultra-structural features, such as lamellar bodies that produce lung surfactant. A549 cells are also a pulmonary epithelial cell model for drug metabolism (6). The phospholipids produced, which are major components of surfactant, are similar to those generated by type II pneumocytes (7). A549 cells typically grow in confluent monolayers.

H441 is a human lung adenocarcinoma epithelial cell line used as a model of Clara cells. Clara cells are similar to type II pneumocytes but are located in the bronchi, where they also produce lung surfactant (8). H441 cells signal and respond to mitogenic growth factors in a manner similar to 
type II pneumocytes (9). These cell lines have previously been used in research studies to assess the link between insulin and SFTPs $(10,11)$.

Lung surfactant is a mixture of phospholipids and proteins that reduces surface tension. Without lung surfactant, the alveoli would collapse due to rapid changes in air pressure. Lung surfactant also controls innate immunity. Surfactant contains 4 proteins that are crucial for its function. SFTPA (SFTPA1 and SFTPA2) and SFTPD are large, hydrophilic proteins that mediate host immunity and are both members of the collectin family (12). The SFTPA monomers vary between 26 and $35 \mathrm{kDa}$ in size, and the SFTPD monomer is $43 \mathrm{kDa}$ in size (13). SFTPA and SFTPD multimers bind to bacterial and fungal cells to suppress growth, stimulate cell lysis and promote phagocytosis (14-16). Both proteins also bind to $\operatorname{IgE}$ and lower allergic hypersensitivity (17). SFTPA and SFTPD are also involved in the removal of apoptotic cells (18), and both proteins inhibit the proliferation of T cells $(19,20)$. SFTPB and SFTPC are small, hydrophobic proteins. SFTPB is a $17-\mathrm{kDa}$ homodimer, with a biophysical function. SFTPB facilitates surfactant adsorption on the surface of the alveoli, and surfactant film would not be functional without SFTPB (21-23). In infants, the lack of SFTPB is associated with SFTPC deficiency and leads to fatal respiratory failure (24). SFTPC is the smallest $(4 \mathrm{kDa})$ and most abundant $(65 \%)$ surfactantassociated protein. SFTPC is an integral membrane protein with recent evolutionary derivation. SFTPC has been identified only in the lung tissue of mammals $(22,25)$. The proper formation of mature SFTPC from its pro-protein depends on the presence of functional SFTPB $(26,27)$. SFTPC binds bacterial lipopolysaccharide to suppress inflammation (13). SFTPC (and SFTPA) also stimulates cells to reabsorb surfactant phospholipids.

The mechanisms underlying the regulation of SFTPs remain elusive; however, regulation at the transcriptional level has been demonstrated. Thyroid transcription factor 1 (TTF-1/Nkx2.1) increases the transcription of SFTPs through interactions with their promoters (28). TTF-1 and hepatocyte nuclear factor (HNF)-3 $\beta$ have been implicated in the induction of SFTP expression $(29,30)$. Furthermore, glucocorticoid and thyroid hormones have been shown to promote surfactant synthesis and protein production through increased cAMP levels $(28,31,32)$. Nitric oxide can inhibit the transcription of SFTPB (33). Little is known about the regulation of SFTP translation in vivo. Glucocorticoids can affect the stability of SFTP mRNA (28), and SFTPA translation can be affected through specific miRNAs (34).

In this study, we attempted to characterize SFTPs in the A549 and H441 cell lines and lung tissue. We examined the combined effect of corticosteroids (dexamethasone was added to stimulate gene expression) and insulin treatment on SFTP expression. Furthermore, we demonstrate the effects of insulin on SFTPs in patients with lung adenocarcinoma undergoing lung resection. The sufficient production of lung surfactant is important in these patients since it directly affects breathing, which is seriously impaired following lung resection. The results obtained in this study contribute to a better understanding of surfactant biology and reveal the limitations of the use of the A549 and H441 cell lines, as it is crucial to know the constraints before selecting a proper in vitro model.

\section{Materials and methods}

Cell lines. The A549 and $\mathrm{H} 441$ cells were obtained from the American Type Culture Collection (ATCC; Rockville, MD, USA). The cells were cultivated in medium (A549, DMEM; H441, RPMI-1640) enriched with 10\% fetal calf serum (FCS), $50 \mathrm{U} / \mathrm{ml}$ penicillin $\mathrm{G}$, and $50 \mu \mathrm{g} / \mathrm{ml}$ streptomycin sulfate at $37^{\circ} \mathrm{C}$ in a humidified atmosphere with $5 \% \mathrm{CO}_{2}$. The cells were grown to $85 \%$ (A549) or 70\% (H441) confluence, washed once with $1 \mathrm{X}$ PBS, and placed in serum-free medium for $24 \mathrm{~h}$ prior to the experiment. Insulin (Humulin; Eli Lilly, Prague, Czech Republic) diluted in water was added to the cultures.

To determine the optimal dosage of insulin, we considered published experiments $(10,11,35)$ and performed optimization experiments, examining serial dilutions of insulin from 2,500 to $0.25 \mathrm{ng} / \mathrm{ml}$. Only the 2 highest concentrations of insulin exhibited a reproducible impact on SFTP transcription. Thus, we used an insulin concentration of $2,500 \mathrm{ng} / \mathrm{ml}$ in the medium in the subsequent experiments.

We also optimized the duration of the insulin treatment $(1,3,6,9,12,24$ and $48 \mathrm{~h})$, using 2,500 $\mathrm{ng} / \mathrm{ml}$ insulin in the medium. The results following exposure to insulin for periods shorter than $24 \mathrm{~h}$ varied greatly, and we did not consider these results as reliable. Thus, we used a 48-h exposure in the subsequent experiments, as the effect of insulin was generally stronger after $48 \mathrm{~h}$ than after $24 \mathrm{~h}$.

Surprisingly, the mRNA levels of some SFTPs were generally low in the cultured cells. Therefore, we added dexamethasone (corticosteroid) to stimulate mRNA expression. Corticosteroids are typically used to stimulate fetal lung maturation in cases of premature labor. Based on previous studies, we used the recommended concentration of $10^{-7} \mathrm{M}$ dexamethasone (Sigma-Aldrich, St. Louis, MO, USA) in the media $(36,37)$. Dexamethasone was diluted in ethanol.

Lung tissue samples. Samples were obtained from 10 patients undergoing lung resection. Written informed consent for the scientific use of biological material was obtained from all patients in accordance with the requirements of the institutional ethics committee. Patient cohorts were divided into 2 groups with and without insulin administration. Insulin was administered to maintain glycemia levels between $6.0-8.0 \mathrm{mmol} / \mathrm{l}$ during surgery. The glycemia level was assessed every hour. The infusion of $10 \%$ glucose was administered at approximately $50 \mathrm{ml} / \mathrm{h}$, and the glucose uptake was maintained at $1 \mathrm{mg} / \mathrm{kg} /$ min. Surgical resection was performed under general anesthesia, with additional epidural anesthesia or paravertebral block. The details and insulin dosage for each patient are presented in Table I. After removal, the lung tissue samples were immediately stored in RNAlater (Ambion, Austin, TX, USA) according to the manufacturer's instructions until total RNA or proteins were isolated. The samples were homogenized using a MagNA Lyser Instrument (Roche Diagnostics, Mannheim, Germany).

RNA isolation. The cells were washed twice with ice-cold 1X PBS, harvested, and subsequently total RNA was isolated using an RNeasy kit (Qiagen, Hilden, Germany) according to the standard protocol. The RNA concentration was determined spectrophotometrically (NanoDrop ND-1000; NanoDrop Technologies, Wilmington, DE, USA). 
Table I. Details of patients with adenocarcinoma.

\begin{tabular}{lccc}
\hline Patients & Gender & $\begin{array}{c}\text { Age } \\
(\text { mean } \pm \text { SD })\end{array}$ & $\begin{array}{c}\text { Insulin dose } \\
(I U, \text { mean } \pm \text { SD })\end{array}$ \\
\hline Untreated & $M(n=2), F(n=3)$ & $61.6 \pm 6.6$ & 0 \\
Treated & $M(n=2), F(n=3)$ & $67.0 \pm 6.4$ & $22.0 \pm 6.6$ \\
\hline
\end{tabular}

Patient details include gender, age, and the total dose of insulin received before the healthy lung tissue surrounding the tumor was removed. M, males; F, females.

Reverse transcription. cDNA was synthesized from $2 \mu \mathrm{g}$ of RNA using a Superscript II reverse transcriptase (Invitrogen, Carlsbad, CA, USA) and oligo(dT) primers (Sigma-Aldrich), according to the manufacturer's instructions. The RNasin RNase Inhibitor (Promega, Mannheim, Germany) was added.

qPCR. The TaqMan ${ }^{\circledR}$ Gene Expression Master Mix was used together with TaqMan ${ }^{\circledR}$ Gene Expression Assays: SFTPA1, Hs01921510_s1; SFTPA2, Hs00359837_m1; SFTPB, Hs00167036_m1; SFTPC, Hs00161628_m1; SFTPD, Hs00358340_m1 (Applied Biosystems, Foster City, USA). In each reaction, $100 \mathrm{ng}$ of cDNA was used, and a human GAPDH endogenous control (Applied Biosystems) was added. GAPDH was selected as a reference gene, as GAPDH has shown a stringent association with lung cells and demonstrates the least variation out of the 7 common reference genes (38). The PCR reaction was performed in 96-well plates (25 $\mu 1$ per well) on a 7500 Real-Time PCR System (Applied Biosystems). The following temperature settings were used: step 1 at $50^{\circ} \mathrm{C}$ for $2 \mathrm{~min}$, step 2 at $95^{\circ} \mathrm{C}$ for $10 \mathrm{~min}$, step 3 at $95^{\circ} \mathrm{C}$ for $15 \mathrm{sec}$, and step 4 at $60^{\circ} \mathrm{C}$ for $1 \mathrm{~min}$. Steps 3 and 4 were repeated 40 times. The expression data obtained from the cell line samples were calculated using the $\Delta \Delta \mathrm{Ct}$ method and expressed as relative units. The data obtained from the lung tissue samples were calculated using the $\Delta \mathrm{Ct}$ method.

Protein isolation. The cells were washed twice with ice-cold $1 \mathrm{X}$ PBS, harvested, lysed in $250 \mu$ l RIPA lysis buffer $[150 \mathrm{mM}$ sodium chloride, $1.0 \%$ NP-40, $0.5 \%$ sodium deoxycholate, $0.1 \%$ SDS, $50 \mathrm{mM}$ Tris ( $\mathrm{pH} 8.0$ ), Complete Protease Inhibitor Cocktail Tablets (Roche Diagnostics), PhosSTOP Phosphatase Inhibitor Cocktail Tablets (Roche Diagnostics)], and sonicated. The protein concentration was determined using the BCA Protein assay (Pierce Biotechnology, Rockford, IL, USA). The samples were subsequently diluted with RIPA buffer to a final concentration of $0.95 \mu \mathrm{g} / \mu \mathrm{l}$ and mixed with 2X Laemmli buffer [0.125 M Tris (pH 6.8), 20\% glycerol, 4\% SDS, $5 \% \beta$-mercaptoethanol and $0.005 \%$ bromophenol blue]. The mixture was boiled at $100^{\circ} \mathrm{C}$ for $5 \mathrm{~min}$ and cooled before loading onto the electrophoresis gel.

SDS-PAGE and western blot analysis. Each cell line sample contained $24 \mu \mathrm{g}$ of total protein and each lung tissue sample contained $4 \mu \mathrm{g}$ of total protein, which was loaded into a $12 \%$ Precise Protein Gel for SDS-PAGE (Pierce Biotechnology). The Amersham Full-Range Molecular Weight Marker RPN800E
(GE Healthcare, Little Chalfont, UK) was used. SDS-PAGE was performed according to the manufacturer's instructions using the Mini-PROTEAN 3 system (Bio-Rad Laboratories, Hercules, CA, USA). The proteins were electrophoresed in TrisHEPES-SDS buffer at $80 \mathrm{~V}$ for $70 \mathrm{~min}$. The primary antibodies were diluted in 5\% BSA (PAN-Biotech, Aidenbach, Germany) and applied overnight at $4^{\circ} \mathrm{C}$. The secondary antibodies were diluted in $1 \mathrm{X}$ TBS buffer containing $0.05 \%$ Tween-20 (SigmaAldrich, St. Louis, MO, USA) and 5\% non-fat milk. The blots were developed using an Immun-Star WesternC kit (Bio-Rad Laboratories) according to the manufacturer's instructions. The images were captured using a G:Box Bio-Imaging System (Syngene, Frederick, MD, USA).

Antibodies. The following primary antibodies were used: mouse monoclonal [HYB 238-04] antibody to surfactant protein A (AB51891; Abcam, Cambridge, UK; dilution 1:200); rabbit anti-human prosurfactant protein B polyclonal antibody (AB3430; Millipore, Temecula, CA, USA; dilution 1:2,000); rabbit anti-prosurfactant protein $\mathrm{C}$ polyclonal antibody (AB3786; Millipore; 1:3,000); mouse monoclonal [12G5] to surfactant protein D (AB17781; Abcam; dilution 1:5,000); and GAPDH (14C10) rabbit monoclonal antibody (\#2118; Cell Signaling Technology, Danvers, MA, USA; dilution 1:1,000). The following secondary antibodies were used: rabbit $\mathrm{IgG}$ secondary antibody, H\&L (AB6721; Abcam, dilution 1:5,000); and anti-mouse IgG (whole molecule) peroxidase conjugate (A9044; Sigma-Aldrich; dilution 1:5,000).

Statistical analysis. The cells were cultured in biological triplicates and each sample was loaded into 3 wells of qPCR plates. The results were transformed using a common logarithm, and based on the results of the f-test, two-sample Student's t-tests with equal or unequal variance were applied. The P-value was set to 0.05 . The analysis compared samples cultured with insulin or dexamethasone to the control samples. The samples cultured with both insulin and dexamethasone were compared to the samples cultured with dexamethasone only. The patients were divided into 2 groups of 5 patients, and the results were analyzed in a similar manner.

\section{Results}

The regulation of surfactant-associated protein expression was investigated using bronchiolar-epithelial cells (H441) and adenocarcinoma-derived alveolar epithelial cells (A549). The cells were exposed to insulin $(2,500 \mathrm{ng} / \mathrm{ml})$ and dexamethasone $\left(10^{-7} \mathrm{M}\right)$, and the expression of SFTPs was determined using qPCR. The proof of principle was tested on a small group $(5+5)$ of lung adenocarcinoma patients. Insulin was administered to these patients to maintain normoglycemia during surgery. Lung samples were obtained from the healthy tissue surrounding the tumor.

SFTPA1 and SFTPA2. No changes in the transcription of the SFTPA1 and SFTPA2 genes were observed in the H441 cells following insulin treatment for $48 \mathrm{~h}$. Dexamethasone treatment decreased SFTPA1 and SFTPA 2 mRNA production by approximately $50 \%$. Surprisingly, the cells that received the combined dexamethasone and insulin treatment showed an 


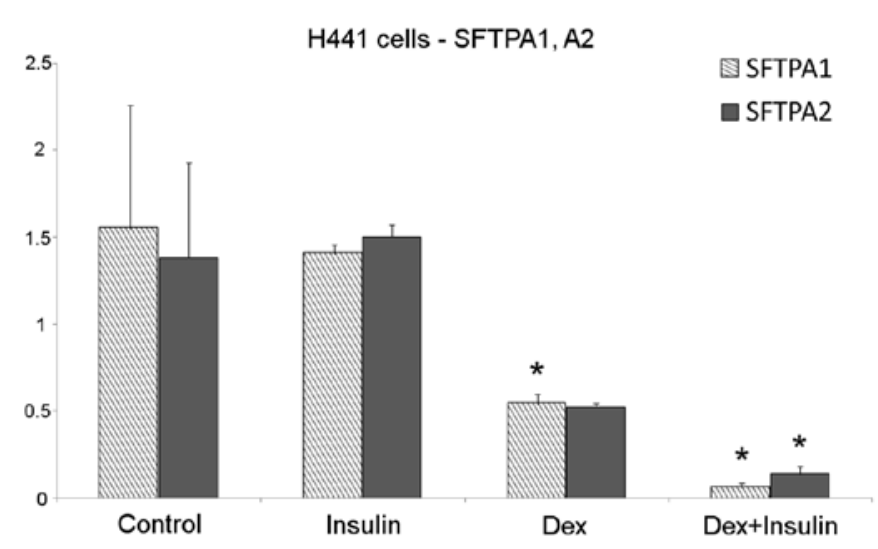

Figure 1. Relative changes in the expression of SFTPA1 and SFTPA2 in H441 cells after $48 \mathrm{~h}$ of treatment. The cells were untreated (control) or treated with $2,500 \mathrm{ng} / \mathrm{ml}$ insulin, $10^{-7} \mathrm{M}$ dexamethasone (Dex), or both for $48 \mathrm{~h}$. The mRNA was extracted, reverse transcribed and quantified using TaqMan-based realtime PCR. In A549 cells, no SFTPA1 and STPA2 mRNA was detected. An asterisk $(*)$ indicates a statistically significant difference from the untreated control (located above the insulin or Dex sample) or Dex (located above the Dex + insulin sample) at $\mathrm{p}<0.05$. SFTP, pulmonary surfactant-associated protein.

enhanced downregulation of SFTPA1 and SFTPA2 compared with the untreated cells (Fig. 1). The SFTPA protein levels were reduced following treatment with dexamethasone or a combination of dexamethasone and insulin. Unlike the mRNA levels, insulin alone also caused a partial reduction in the SFTPA protein level (Fig. 2).

The mRNAs encoding SFTPA1 and SFTPA 2 or the SFTPA protein were not detected in the A549 cell line. In the lungs, the SFTPA 1 mRNA level was reduced by $30 \%$ following treatment with insulin; however, this change was not statistically significant. No change was observed in the SFTPA2 mRNA levels (Fig. 4). The levels of total SFTPA protein varied among the patients; however, no change was observed in response to insulin treatment (Fig. 5).

SFTPB. Significant changes in SFTPB expression were detected in the H441 and A549 cell lines following treatment with insulin or dexamethasone. Treatment with dexamethasone alone increased the level of SFTPB mRNA in both cell lines. The cells treated with a combination of dexamethasone and insulin exhibited a strong inhibition of SFTPB mRNA transcription compared with cells treated with dexamethasone alone. The rapid reduction in the SFTPB mRNA level was observed in the H441 cells treated with insulin alone. Untreated or insulin-treated A549 cells produced only trace amounts of SFTPB mRNA (Fig. 3).

We then focused on the detection of the pro-form of SFTPB, as mature SFTPB is a small molecule $(8 \mathrm{kDa})$ and we were not able to detect it in the cell lines used in the current study. The observed levels of proSFTPB protein were low or barely detectable in the H441 cells (Fig. 2), although the level of SFTPB mRNA was 100,000-fold higher in the H441 cells compared to the A549 cells (data not shown). However, in the A549 cells, an increase in the proSFTPB protein level was observed following treatment with dexamethasone. The combined treatment with dexamethasone and insulin reduced

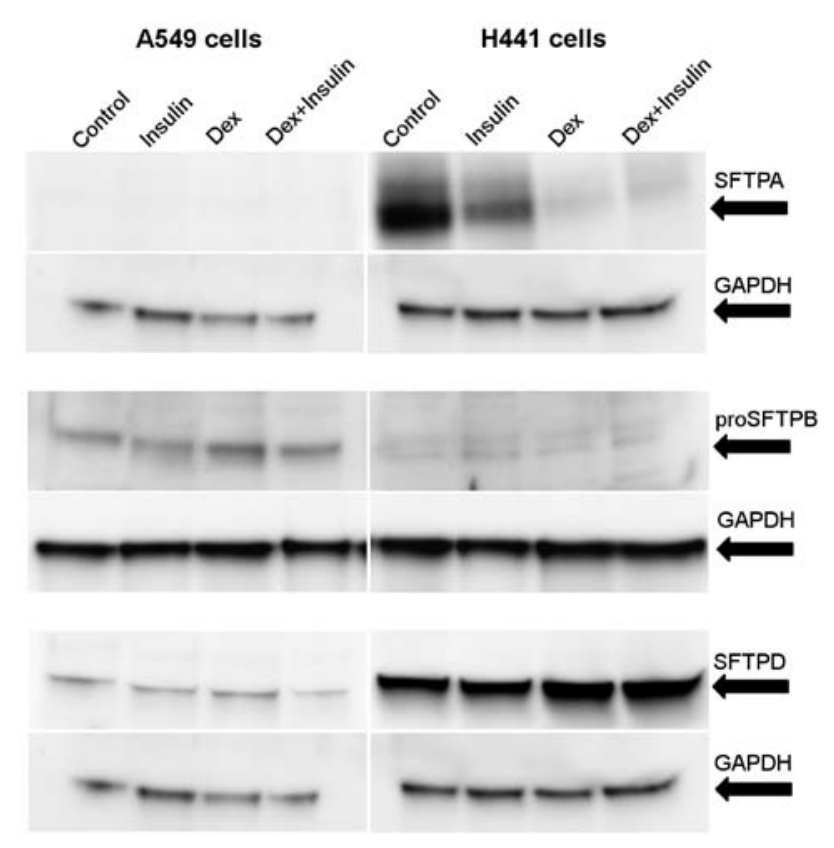

Figure 2. Western blot analysis of A549 and H441 cells after $48 \mathrm{~h}$ of treatment. The cells were untreated (control) or treated with 2,500 ng/ml insulin, $10^{-7} \mathrm{M}$ dexamethasone (Dex), or both for $48 \mathrm{~h}$. The cells were lysed in RIPA buffer, sonicated and loaded onto a polyacrylamide gel $(12 \%)$ for electrophoresis under denaturing conditions. No SFTPA protein was detected in the A549 cells. No SFTPC or proSFTPC protein was detected in A549 or H441 cells. SFTP, pulmonary surfactant-associated protein.

the proSFTPB protein level. In the patients, statistically significant lower levels of SFTB mRNA (50\%) and protein (partially) were observed in the insulin-treated patients compared with the control group (Figs. 4 and 5).

SFTPC. A significant upregulation in the SFTPC mRNA level following treatment with dexamethasone was observed in the H441 cells. In these cells, a significant reduction in the dexamethasone-induced expression of SFTPC was observed following $48 \mathrm{~h}$ of insulin co-treatment.

The A549 cells did not respond to insulin treatment, and a minimum response to the dexamethasone treatment was observed (Fig. 3). As both cell lines produced only a trace amount of SFTPC mRNA, we were unable to detect any SFTPC or proSFTPC protein expression. A statistically significant reduction (45\%) in SFTC mRNA expression in the insulin-treated patients was observed (Fig. 4), and the level of proSFTPC protein was also reduced in this group (Fig. 5).

SFTPD. Both H441 and A549 cells produced SFTPD, which was further stimulated following treatment with dexamethasone. Treatment with insulin inhibited the normal or dexamethasone-induced transcription of SFTPD mRNA in the H441 cells, but not in the A549 cells (Fig. 3). In the A549 cells, the combined treatment of dexamethasone and insulin reduced the SFTPD protein levels; however, in the H441 cells, dexamethasone treatment, with or without insulin, elevated SFTPD protein levels (Fig. 2).

These results provide evidence for the differential regulation of mature protein through dexamethasone and insulin. In the clinical samples, a 50\% reduction in SFTPD mRNA levels 

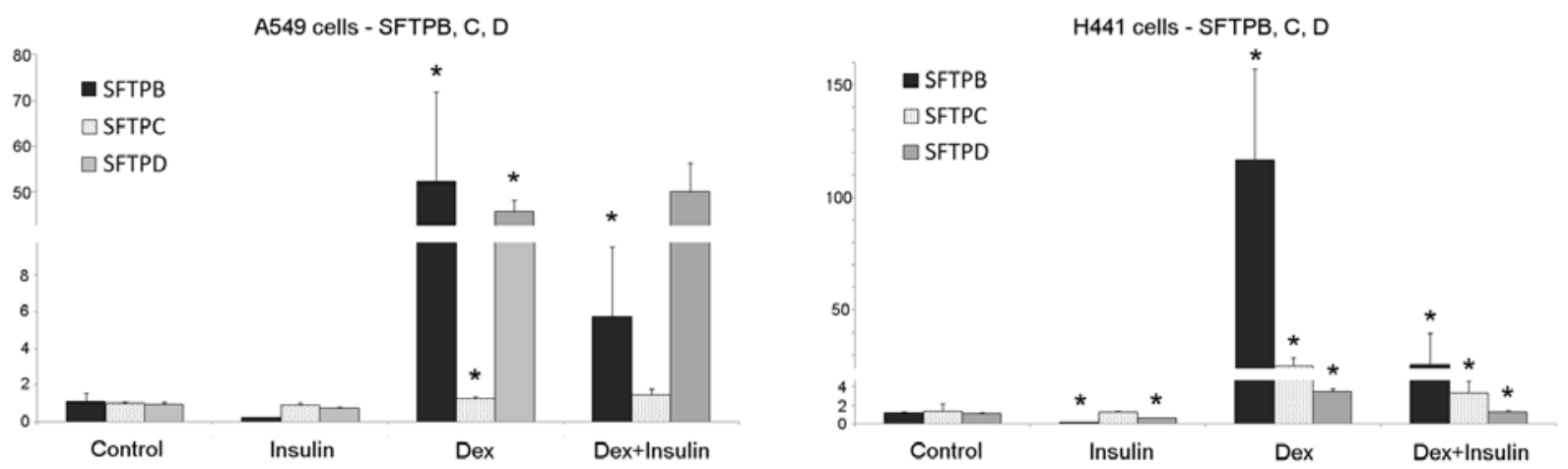

Figure 3. Relative changes in SFTPB, SFTPC and SFTPD mRNA levels in A549 (left) and H441 (right) cells after $48 \mathrm{~h}$ of treatment. The cells were untreated (control) or treated with $2,500 \mathrm{ng} / \mathrm{ml}$ insulin, $10^{-7} \mathrm{M}$ dexamethasone (Dex), or both for $48 \mathrm{~h}$. The mRNA was extracted, reverse transcribed and quantified using TaqMan-based real-time PCR. An asterisk (*) indicates a statistically significant difference from the untreated control (located above the insulin or Dex sample) or Dex (located above the Dex + insulin sample) at $\mathrm{p}<0.05$. SFTP, pulmonary surfactant-associated protein.

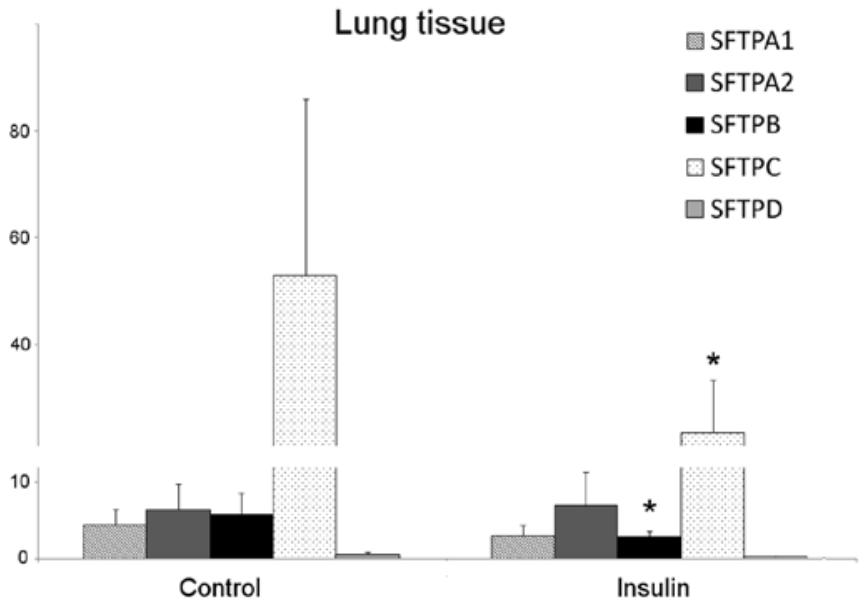

Figure 4. Relative changes in SFTPA1 and A2, SFTPB, SFTPC and SFTPD mRNA levels in lung tissue samples. Half of the patients were treated with insulin to maintain normoglycemia. The mRNA was extracted; reverse transcribed and quantified using TaqMan-based real-time PCR. An asterisk (*) indicates a statistically significant difference from the untreated control at $\mathrm{p}<0.05$. SFTP, pulmonary surfactant-associated protein.

following treatment with insulin was observed; however, this result was not statistically significant (Fig. 4). No difference in the SFTPD protein expression was observed (Fig. 5). These results are summarized in Table II.

Differences in mRNA levels. Surprisingly, significant differences in the mRNA levels were observed between the lung tissue samples and the cell lines using the $\Delta \Delta \mathrm{Ct}$ method (GAPDH was used as a reference gene in both cell lines and lung tissue). The expression of SFTPA1 and A 2 in the control samples was 100- and 200-fold lower, respectively in the H441 cell line. No SFTPA mRNA expression was detected in the A549 cell line. The SFTPB mRNA level in the H441 cells was approximately 2,000-fold lower than that in the lung tissue, and approximately 10,000,000-fold lower in the A549 cells. SFTPC mRNA was the most abundant mRNA in the lung tissue, but in both cell lines only trace amounts were detected, with approximately 10,000,000-fold lower concentrations compared to the lung tissue. The SFTPD mRNA concentra-

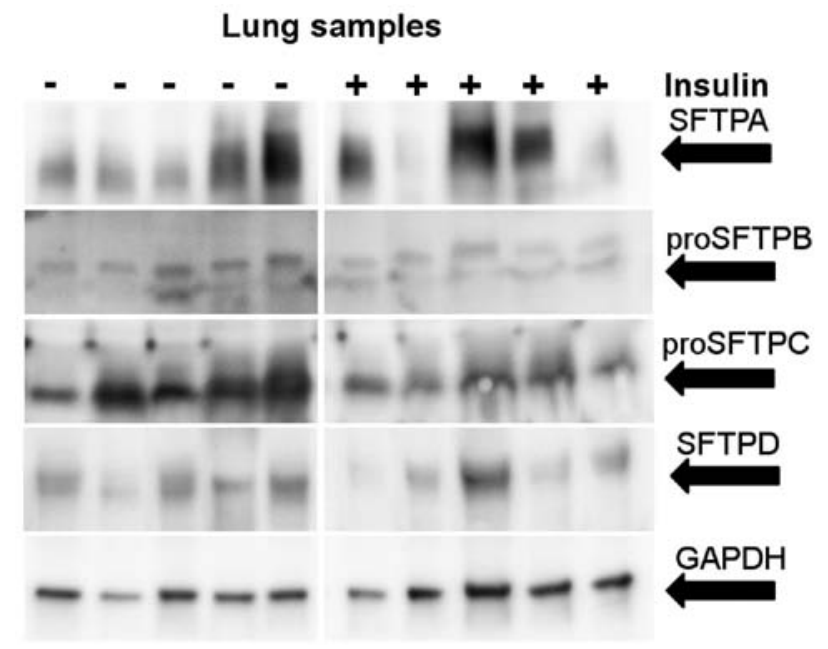

Figure 5. Western blot analysis of lung tissue samples. Half of the patients were treated with insulin to maintain normoglycemia. The tissue was homogenized, lysed in RIPA buffer, sonicated and loaded onto a polyacrylamide gel $(12 \%)$ for electrophoresis under denaturing conditions. SFTP, pulmonary surfactant-associated protein.

tion was 10,000-fold lower in the cell lines compared to the lung tissue.

\section{Discussion}

The aim of this study was to examine the effects of insulin on the transcription and translation of SFTPs in lung surfactantproducing cells. Two cell lines, A549 and H441, are commonly used as models of surfactant-producing cells. Surprisingly, the 2 cell lines used in the current study differed in several biological aspects, particularly as regards the production of SFTPs. Moreover, there was a significant difference in SFTP mRNA levels in these cell lines compared to those in the lung tissue samples, making it difficult to generalize the complex role of insulin and dexamethasone in primary surfactant-producing lung cells.

Specifically, expression analysis revealed the significantly reduced production of SFTPA mRNA in the H441 cells and no production of SFTPA mRNA in the A549 cells. In addition, the 
Table II. Regulation of mRNA/protein levels of SFTPs in A549 and H441 cells following treatment with insulin, dexamethasone or a combination of both for $48 \mathrm{~h}$.

\begin{tabular}{|c|c|c|c|c|c|c|}
\hline Cell line & Treatment & SFTPA $1^{\mathrm{a}}$ & SFTPA2 ${ }^{a}$ & SFTPB $^{b}$ & SFTPC & SFTPD \\
\hline \multirow[t]{3}{*}{ A549 } & Insulin & $-/-$ & $-/-$ & $\leftrightarrow / \leftrightarrow$ & $\leftrightarrow /-$ & $\leftrightarrow / \leftrightarrow$ \\
\hline & Dex & $-/-$ & $-/-$ & $\uparrow \uparrow \uparrow \uparrow / \uparrow$ & $\leftrightarrow /-$ & $\uparrow \uparrow \uparrow / \leftrightarrow$ \\
\hline & Dex + insulin & $-/-$ & $-/-$ & $\uparrow / \leftrightarrow$ & $\leftrightarrow / \uparrow$ & $\uparrow \uparrow \uparrow / \downarrow$ \\
\hline \multirow[t]{3}{*}{ H441 } & Insulin & $\leftrightarrow / \downarrow$ & $\leftrightarrow / \downarrow$ & $\downarrow / \leftrightarrow$ & $\leftrightarrow /-$ & $\downarrow / \leftrightarrow$ \\
\hline & Dex & $\downarrow / \downarrow \downarrow$ & $(\downarrow) / \downarrow \downarrow$ & $\uparrow \uparrow \uparrow / \leftrightarrow$ & $\uparrow \uparrow /-$ & $\uparrow / \uparrow$ \\
\hline & Dex + insulin & $\downarrow \downarrow / \downarrow \downarrow$ & $\downarrow \downarrow / \downarrow \downarrow$ & $\uparrow / \leftrightarrow$ & $\uparrow /-$ & $\leftrightarrow / \uparrow$ \\
\hline
\end{tabular}

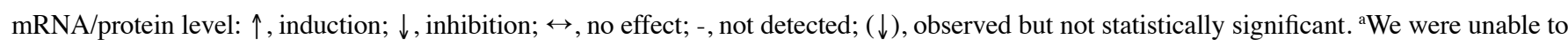
distinguish between the SFTPA1 and SFTPA2 protein isoforms. ${ }^{b} \mathrm{We}$ were unable to detect mature protein; therefore, we focused on the precursor form, proSFTPB. SFTP, pulmonary surfactant-associated protein; Dex, dexamethasone.

SFTPB mRNA level was low in the H441 cells, and only trace amounts were detected in the A549 cells. The levels of SFTPC and SFTPD mRNA were extremely low in both cell lines. The concentration of SFTPC mRNA in the H441 cells was barely detectable, and it was originally considered that the $\mathrm{H} 441$ cells do not synthesize SFTPC mRNA (10). Accordingly, the levels of SFTPC or proSFTPC proteins were below detection limits in the cell lines used in the present study.

To compensate for the low quantity of SFTP mRNA and proteins in these cell lines, dexamethasone was successfully applied to stimulate the transcription of the SFTPB, SFTPC and SFTPD genes. The effects of insulin treatment were accurately measured following treatment with dexamethasone; however, the the relatively large differences in the mRNA levels between the cell lines and lung tissue were maintained.

Our results (Table II) demonstrated that insulin reduced the mRNA transcription of the SFTPB, SFTPC and SFTPD genes in the $\mathrm{H} 441$ cells, but did not alter the transcription of SFTPA1 and SFTPA2, unless used in combination with dexamethasone. Surprisingly, the combination of insulin and dexamethasone significantly reduced the transcription of SFTPA mRNA compared with the effects of dexamethasone alone. In the A549 cells, only SFTPB transcription was downregulated following treatment with insulin. Insulin did not affect SFTPC or SFTPD mRNA levels.

Although the SFTPA mRNA level in the H441 cells was not affected by insulin alone, the level of SFTPA protein was reduced in response to insulin, but not to the extent of the reduction observed with dexamethasone treatment alone. The treatment with dexamethasone alone, or in combination with insulin, induced only trace amounts of the SFTPA protein in the H441 cells. As expected, based on the absence of SFTPA1 or A2 mRNA, no SFTPA protein was detected in the A549 cells. Our results suggested that the stability of the proSFTPB protein was impaired in the $\mathrm{H} 441$ cells. However, the detection of mature SFTPB protein using western blot analysis was not successful, as the mature SFTPB protein had a low molecular weight. In the A549 cells, insulin counteracted the increase in proSFTPB protein levels induced by dexamethasone. This result was consistent with the qPCR results. Therefore, we suggest that the increase in proSFTPB protein levels is a direct consequence of lower SFTPB mRNA production.
Although SFTPD protein was detected in both cell lines, this protein was more abundant in the $\mathrm{H} 441$ cells. This difference was further increased following treatment with dexamethasone. Insulin treatment did not counteract the effects of dexamethasone on the expression of SFTPD protein. We suggest that the stability of the SFTPD protein is greater in the H441 cells since the SFTPD protein level is significantly higher in the $\mathrm{H} 441$ cells than in the A549 cells, despite the fact that both cell lines contain the same amount of SFTPD mRNA.

SFTPB protein levels remained unresponsive to the increased mRNA levels induced by dexamethasone in the A549 cells. This result may reflect a reduction in the stability of this protein in A549 cells. The combination of dexamethasone and insulin treatment exerted inhibitory effects on the expression of SFTPB protein in the A549 cells and SFTPA protein in the $\mathrm{H} 441$ cells.

In contrast to the findings of previous studies concerning the effects of insulin in human fetal lung explants maintained in vitro (39) and $\mathrm{H} 441$ cells (10), we did not observe any inhibitory effects of insulin on the SFTPA1 and SFTPA2 mRNA levels after $48 \mathrm{~h}$ of insulin treatment in the $\mathrm{H} 441$ cells. However, we also observed a reduction in SFTPB mRNA levels in the H441 cells, consistent with the findings of the aforementioned studies. We observed a reduction in SFTPC protein levels in the $\mathrm{H} 441$ cells following treatment with dexamethasone and insulin. This result was not observed in the A549 cells. In human fetal lung explants, no significant effect on SFTPC mRNA levels was observed (39).

In this study, we demonstrate that dexamethasone differentially upregulates the transcription of SFTPB, SFTPC and SFTPD in the H441 and A549 cell lines, suggesting that this process involves cell context-dependent transcriptional machinery. The transcription of SFTPA1 and SFTPA2 mRNA was enhanced following treatment with dexamethasone in the H441 cells. In non-human primate fetal lung cells, dexamethasone treatment has also been shown to reduce the levels of SFTPA mRNA (authors did not distinguish the 2 isoforms), although the sensitivity to dexamethasone treatment was reduced as the tissue matured (40). In the present study, the H441 cells showed typical characteristics of immature lung tissue. According to Kumar and Snyder (35), treatment with dexamethasone reduced the SFTPA1 mRNA level by $60 \%$, 
and treatment with insulin reduced the SFTPA1 mRNA level by $40 \%$ in the $\mathrm{H} 441$ cells. Moreover, dexamethasone and insulin co-treatment did not affect the SFTPA2 mRNA levels. In this study, we confirmed that dexamethasone reduces SFTPA1 transcription, and we observed an equivalent reduction (approximately 50\%) in SFTPA2 transcription. Moreover, in the study by Kumar and Snyder, using human fetal lung explants, treatment with dexamethasone or insulin reduced the SFTPA1 and the SFTPA2 mRNA level to the same degree (35). In the present study, we did not detect any significant effects of insulin treatment on SFTPA1 and SFTPA2 mRNA levels, unless insulin was combined with dexamethasone.

The stimulatory effect of dexamethasone on the SFTPB and SFTPC transcription has been previously established (41). This effect may reflect the increased transcription and SFTPB mRNA stabilization, in addition to the increased transcription and disputable effect on SFTPC mRNA stabilization (42-46). Previous studies have shown that SFTPD transcription is enhanced by treatment with dexamethasone, although there are no data concerning the effect of dexamethasone on the stability of SFTPD mRNA (47). In our study, surprisingly, the A549 cells responded to dexamethasone treatment and displayed an increase in the production of SFTPC mRNA. The H441 cells exhibited a slight increase in the production of SFTPD mRNA.

Two factors challenge the suitability of the A549 and H441 cell lines as models for lung surfactant-producing cells, type II pneumocytes and Clara cells: low levels of SFTP mRNA in the A549 and H441 cells compared with the lung tissue and the differences between the 2 cell lines, which were expected to exhibit similar SFTP production. We suggest that the use of A549 and H441 cells is limited, and no solid conclusions concerning SFTPs should be based on experiments performed only on these cell lines.

Therefore, our study demonstrates the possibility of measuring the effects of insulin on SFTP expression in patients with lung adenocarcinoma. However, insulin is only administered to maintain normoglycemia, which is disrupted through stress as a result of surgery. Therefore, it is only possible to examine the effects of insulin in a range of hours and not days. In this study, we focused on insulin- and dexamethasoneinduced changes in the expression of SFTPs. We demonstrate that insulin reduces the transcription of SFTPB, SFTPC and SFTPD in H441 cells. In the A549 cells, only SFTPB transcription was downregulated following treatment with insulin. The expression of SFTPA1 and SFTPA2 was only detected in the H441 cells, although insulin did not affect their mRNA levels. Insulin significantly reduced the protein levels of SFTPA in the H441 cells. The inhibitory effect of insulin on proSFTPB protein levels was observed in the A549 cells. Dexamethasone upregulated the transcription of SFTPB, SFTPC and SFTPD genes in the A549 and H441 cells. Dexamethasone downregulated the transcription of the SFTPA1 and SFTPA2 genes in the H441 cells. However, the differences between the A549 and H441 cells and the generally low levels of SFTP mRNA compared with the lung tissue, challenge the suitability of these cell lines as models of surfactant-producing cells. We recommend studying the effects of insulin on SFTPs in patients, demonstrating the feasibility of these experiments using tissues samples from patients with lung adenocarcinoma during lung resection. We suggest that the use of these samples may generate reliable results when the use of cell line models is disputable.

\section{Acknowledgements}

This study was supported through funding from the Czech Science Foundation (GACR P302/12/G157) and the Ministry of Education, Youth and Sports of the Czech Republic (MSM 0021622430).

\section{References}

1. Bland D, Fankhanel Y, Langford E, et al: Intensive versus modified conventional control of blood glucose level in medical intensive care patients: a pilot study. Am J Crit Care 14: 370-376, 2005.

2. Finfer S, Chittock D, Su S, et al: Intensive versus conventional glucose control in critically ill patients. N Engl J Med 360: 1283-1297, 2009.

3. Van den Berghe G, Wouters P, Weekers F, et al: Intensive insulin therapy in the critically ill patients. N Engl J Med 345: 1359-1367, 2001.

4. Van den Berghe G: How does blood glucose control with insulin save lives in intensive care? J Clin Invest 114: 1187-1195, 2004.

5. Van den Berghe G, Schetz M, Vlasselaers D, et al: Clinical review: Intensive insulin therapy in critically ill patients: NICESUGAR or Leuven blood glucose target? J Clin Endocrinol Metab 94: 3163-3170, 2009.

6. Foster K, Oster C, Mayer M, Avery M and Audus K: Characterization of the A549 cell line as a type II pulmonary epithelial cell model for drug metabolism. Exp Cell Res 243: 359-366, 1998.

7. Nardone LL and Andrews SB: Cell line A549 as a model of the type II pneumocyte. Phospholipid biosynthesis from native and organometallic precursors. Biochim Biophys Acta 573: 276-295, 1979.

8. Ladenburger A, Seehase M, Kramer BW, et al: Glucocorticoids potentiate IL-6-induced SP-B expression in H441 cells by enhancing the JAK-STAT signaling pathway. Am J Physiol Lung Cell Mol Physiol 299: L578-L584, 2010.

9. Chess PR, Ryan RM and Finkelstein JN: H441 pulmonary epithelial cell mitogenic effects and signaling pathways in response to HGF and TGF-alpha. Exp Lung Res 24: 27-39, 1998.

10. Miakotina OL, Dekowski SA and Snyder JM: Insulin inhibits surfactant protein A and B gene expression in the H441 cell line. Biochim Biophys Acta 1442: 60-70, 1998.

11. Miakotina OL, Goss KL and Snyder JM: Insulin utilizes the PI 3-kinase pathway to inhibit SP-A gene expression in lung epithelial cells. Respir Res 3: 27, 2002.

12. Holmskov U, Malhotra R, Sim R and Jensenius J: Collectins: collagenous C-type lectins of the innate immune defense system. Immunol Today 15: 67-74, 1994.

13. Chaby R, Garcia-Verdugo I, Espinassous Q and Augusto LA: Interactions between LPS and lung surfactant proteins. J Endotoxin Res 11: 181-185, 2005.

14. McCormack F, Gibbons R, Ward S, Kuzmenko A, Wu H and Deepe GJ: Macrophage-independent fungicidal action of the pulmonary collectins. J Biol Chem 278: 36250-36256, 2003.

15. Wu H, Kuzmenko A, Wan S, et al: Surfactant proteins A and D inhibit the growth of Gram-negative bacteria by increasing membrane permeability. J Clin Invest 111: 1589-1602, 2003.

16. van Iwaarden F, Welmers B, Verhoef J, Haagsman H and van Golde L: Pulmonary surfactant protein A enhances the hostdefense mechanism of rat alveolar macrophages. Am J Respir Cell Mol Biol 2: 91-98, 1990.

17. Deb R, Shakib F, Reid K and Clark H: Major house dust mite allergens Dermatophagoides pteronyssinus 1 and Dermatophagoides farinae 1 degrade and inactivate lung surfactant proteins A and D. J Biol Chem 282: 36808-36819, 2007.

18. Vandivier RW, Ogden CA, Fadok VA, et al: Role of surfactant proteins $\mathrm{A}, \mathrm{D}$, and $\mathrm{Clq}$ in the clearance of apoptotic cells in vivo and in vitro: calreticulin and CD91 as a common collectin receptor complex. J Immunol 169: 3978-3986, 2002.

19. Borron P, Veldhuizen RA, Lewis JF, et al: Surfactant associated protein-A inhibits human lymphocyte proliferation and IL-2 production. Am J Respir Cell Mol Biol 15: 115-121, 1996. 
20. Borron P, McCormack FX, Elhalwagi BM, et al: Surfactant protein A inhibits $\mathrm{T}$ cell proliferation via its collagen-like tail and a 210-kDa receptor. Am J Physiol 275: L679-L686, 1998.

21. Nogee L: Genetic mechanisms of surfactant deficiency. Biol Neonate 85: 314-318, 2004

22. Serrano AG and Pérez-Gil J: Protein-lipid interactions and surface activity in the pulmonary surfactant system. Chem Phys Lipids 141: 105-118, 2006.

23. Cruz A, Worthman LA, Serrano AG, Casals C, Keough KM and Pérez-Gil J: Microstructure and dynamic surface properties of surfactant protein SP-B/dipalmitoylphosphatidylcholine interfacial films spread from lipid-protein bilayers. Eur Biophys J 29: 204-213, 2000.

24. Beers M, Hamvas A, Moxley M, et al: Pulmonary surfactant metabolism in infants lacking surfactant protein B. Am J Respir Cell Mol Biol 22: 380-391, 2000

25. Besnard V, Wert SE, Kaestner KH and Whitsett JA: Stagespecific regulation of respiratory epithelial cell differentiation by Foxa1. Am J Physiol Lung Cell Mol Physiol 289: L750-L759, 2005.

26. Weaver TE and Conkright JJ: Function of surfactant proteins B and C. Annu Rev Physiol 63: 555-578, 2001.

27. Clark JC, Wert SE, Bachurski CJ, et al: Targeted disruption of the surfactant protein B gene disrupts surfactant homeostasis, causing respiratory failure in newborn mice. Proc Natl Acad Sci USA 92: 7794-7798, 1995.

28. Boggaram V: Regulation of lung surfactant protein gene expression. Front Biosci 8: d751-d764, 2003.

29. Zhou L, Lim L, Costa RH and Whitsett JA: Thyroid transcription factor-1, hepatocyte nuclear factor-3beta, surfactant protein $B$ $\mathrm{C}$, and Clara cell secretory protein in developing mouse lung. $\mathrm{J}$ Histochem Cytochem 44: 1183-1193, 1996.

30. Stahlman MT, Gray ME and Whitsett JA: Temporal-spatial distribution of hepatocyte nuclear factor-3beta in developing human lung and other foregut derivatives. J Histochem Cytochem 46: 955-962, 1998.

31. Mendelson CR: Endocrinology of the Lung: Development and Surfactant Synthesis. Humana Press, Totowa, New Jersey, 2000.

32. Mendelson CR and Boggaram V: Regulation of pulmonary surfactant protein synthesis in fetal lung: a major role of glucocorticoids and cyclic AMP. Trends Endocrinol Metab 1: 20-25, 1989.

33. Boggaram V, Chandru H, Gottipati KR, Thakur V, Das A and Berhane K: Transcriptional regulation of SP-B gene expression by nitric oxide in H441 lung epithelial cells. Am J Physiol Lung Cell Mol Physiol 299: L252-L262, 2010.

34. Silveyra P and Floros J: Genetic complexity of the human surfactant-associated proteins SP-A1 and SP-A2. Gene, 2012.
35. Kumar A and Snyder J: Differential regulation of SP-A1 and SP-A2 genes by cAMP, glucocorticoids, and insulin. Am J Physiol 274: L177-L185, 1998.

36. Mouhieddine-Gueddiche OB, Pinteur C, Chailley-Heu B, Barlier-Mur AM, Clement A and Bourbon JR: Dexamethasone potentiates keratinocyte growth factor-stimulated SP-A and SP-B gene expression in alveolar epithelial cells. Pediatr Res 53: 231-239, 2003.

37. Salinas D, Sparkman L, Berhane K and Boggaram V: Nitric oxide inhibits surfactant protein $B$ gene expression in lung epithelial cells. Am J Physiol Lung Cell Mol Physiol 285: L1153-L1165, 2003.

38. Liu D, Chen S and Liu H: Choice of endogenous control for gene expression in nonsmall cell lung cancer. Eur Respir J 26: 1002-1008, 2005.

39. Dekowski S and Snyder J: Insulin regulation of messenger ribonucleic acid for the surfactant-associated proteins in human fetal lung in vitro. Endocrinology 131: 669-676, 1992.

40. Seidner S, Smith M and Mendelson C: Developmental and hormonal regulation of SP-A gene expression in baboon fetal lung. Am J Physiol 271: L609-L616, 1996.

41. Liley H, White R, Warr R, Benson B, Hawgood S and Ballard P: Regulation of messenger RNAs for the hydrophobic surfactant proteins in human lung. J Clin Invest 83: 1191-1197, 1989.

42. Boggaram V and Margana R: Developmental and hormonal regulation of surfactant protein $\mathrm{C}$ (SP-C) gene expression in fetal lung. Role of transcription and mRNA stability. J Biol Chem 269: 27767-27772, 1994.

43. Margana R and Boggaram V: Transcription and mRNA stability regulate developmental and hormonal expression of rabbit surfactant protein B gene. Am J Physiol 268: L481-L490, 1995.

44. Huang H, Bi W, Jenkins G and Alcorn J: Glucocorticoid regulation of human pulmonary surfactant protein-B mRNA stability involves the 3'-untranslated region. Am J Respir Cell Mol Biol 38 : 473-482, 2008.

45. Planer B, Ning Y, Kumar S and Ballard P: Transcriptional regulation of surfactant proteins SP-A and SP-B by phorbol ester. Biochim Biophys Acta 1353: 171-179, 1997.

46. Venkatesh V, Iannuzzi D, Ertsey R and Ballard P: Differential glucocorticoid regulation of the pulmonary hydrophobic surfactant proteins SP-B and SP-C. Am J Respir Cell Mol Biol 8: 222-228, 1993

47. Mariencheck W and Crouch E: Modulation of surfactant protein D expression by glucocorticoids in fetal rat lung. Am J Respir Cell Mol Biol 10: 419-429, 1994. 